\title{
Speeding Up COVID-19 Detection Using Shaker-Mill Homogenization and a Direct-to-PCR Workflow
}

Zachary P. Morehouse ${ }^{1,2, * *}$, Caleb M Proctor ${ }^{2,3}$, Gabriella L Ryan ${ }^{2,3}$, Rodney J Nash ${ }^{2,3,4, *}$

1. Michigan State University College of Osteopathic Medicine, East Lansing MI, USA; moreho17@msu.edu (Z.P.M.)

2. Omni International Inc, Kennesaw GA, USA; moreho17@msu.edu (Z.P.M.), cproctor@omniinc.com (C.M.P.), gryan@omni-inc.com (G.L.R.), rnash@omni-inc.com (R.J.N.)

3. Georgia State University, Department of Biology, Atlanta GA, USA; cproctor@omni-inc.com (C.M.P.), gryan@omni-inc.com (G.L.R.), rnash@omni-inc.com (R.J.N.)

4. JeevanBiosciences, Tucker GA, USA; rnash@omni-inc.com (R.J.N.)

* Corresponding Author, rnash@omni-inc.com, tel: +1 770-421-0058

** Co-Corresponding Author, moreho17@msu.edu, tel: +1 813-476-5661 


\begin{abstract}
Accurate and timely testing has become an essential measure in combatting the COVID-19 global pandemic. Currently, polymerase chain reaction (PCR) based assays are the most relied on methods for SARS-CoV-2 detection. This traditional workflow involves a viral RNA extraction from the viral transport media storing nasopharyngeal swabs collected from patients, followed by PCR based detection. While accurate, this methodology is time consuming and resource heavy, causing for delays in receiving results or limited access to testing. Herein, we demonstrate a validated method for SARS-CoV-2 detection from viral transport media using a two-step, direct-to-PCR workflow revolving around shaker-mill homogenization. This method completely bypasses the extraction steps of the traditional workflow, replacing it with 30 seconds of mechanical disruption sufficient to allow for COVID-19 detection with a $96.43 \%$ sensitivity and $100 \%$ specificity when compared to traditional extraction to PCR based methods.
\end{abstract}

\title{
Key Words $(3-10)$ :
}

COVID-19, Virus Detection, Diagnostics, SARS-CoV-2, Coronavirus, Viral Diagnostics, PCR 


\section{Introduction}

Amid the COVID-19 pandemic, the critical need for efficient and cost-effective viral detection methods has been amplified globally [1,2]. Currently, the most relied on method for SARS-CoV-2 detection is a standard viral RNA extraction procedure followed by a polymerase chain reaction (PCR) based assay looking to detect a specific gene product from replicating viruses off patient acquired nasopharyngeal swabs $[2,3,4]$. This methodology has been proven time and time again to be effective in respiratory virus detection from patients actively infected with everything from influenza to SARS-CoV-2 $[3,4,5]$. However, this process of extraction followed by PCR based detection requires significant amounts of chemical reagents, consumable plastics, and laboratory man-hours to complete. These costs of both capital and time, potentially cause delays in patients receiving their results, and the possibility of degradation of viral transcripts within the storage media impairing detection $[6,7]$. Shortages in the supplies required for adequate viral detection and significant delays in patient notifications following nasopharyngeal swab collections have both been attributed to the exponential growth of COVID-19 cases in the United States in June and July of 2020 [8].

Herein, we are proposing a validated methodology for SARS-CoV-2 detection from viral transport media (VTM) storing patient derived nasopharyngeal swabs, which completely bypasses the extraction portion of the classic workflow described above. This novel process employs shaker-mill homogenization to mechanically lyse the virus particles in the transport media, permitting the lysate to be directly transferred into a PCR based assay for detection. Taking a total of 30 seconds of processing prior to the sample being added to PCR detection assays, this method allows patient samples to be processed directly in the viral transport media (VTM) they are stored in following patient sampling, significantly reducing the resources invested and time spent on processing each sample for virus detection.

\section{Materials and Methods}

\subsection{Samples Used for Method Validation}

The samples used for this method validation were obtained via a materials transfer agreement with Emory University's School of Medicine and their Clinical Virology Research Laboratory (Atlanta, GA, USA). These samples were obtained with informed patient consent under and following the protocols approved through the Emory University Institutional Review Board (IRB) (IRB Contract 000001082) and conducted following the rules of the Declaration of Helsinki of 1975.

Each patient provided two nasopharyngeal swab samples. One of the samples was validated as COVID19 positive or negative by the CLIA approved laboratory at Emory University's Clinical Virology Research Laboratory following the traditional, US Centers for Disease Control and Prevention approved extraction and PCR based detection protocols. The second sample from the same patient was then deidentified and labeled as only COVID-19 positive or negative prior to transfer to our laboratory. 58 samples were transferred, 30 confirmed COVID-19 and 28 confirmed COVID-19 positive patient samples. The samples remained stored in their original viral transport media (Fisher, Cat. No. 23-001-726) and collection tube which they were placed in at the time of acquisition and were frozen at $-80^{\circ} \mathrm{C}$ for storage prior to transfer.

\subsection{Patient Sample Processing}


Patient samples stored in viral transport media were thawed in their original collection tubes at $24^{\circ} \mathrm{C}$ for $1 \mathrm{hr}$, or until VTM had completely thawed. The swabs and $1 \mathrm{~mL}$ of VTM were then transferred to a $2 \mathrm{~mL}$ screw cap tube (Omni International Inc., Cat. No. 19-647) and sealed [9]. 24 samples were loaded into the Omni Bead Ruptor Elite (Cat. No. 19-040E) for processing. The samples were run at $4.2 \mathrm{~m} / \mathrm{s}$ for 30 seconds and removed from the device [9]. The samples were permitted to sit for 1 minute after processing to allow for any froth that formed in the tubes during shaker-mill homogenization to settle [9].

\subsection{RT-qPCR Detection of SARS-CoV-2}

$1 \mu \mathrm{L}$ of lysate was transferred into a premixed RT-qPCR plate from the $2 \mathrm{~mL}$ screw cap tube containing the nasopharyngeal swab in VTM following shaker-mill homogenization. The RT-qPCR reaction plate was premixed with New England Biolab's Luna Universal Probe Based One-Step RT-qPCR Kit (Cat. No. E3006) and US CDC approved primers and probes for the nucleocapsid (N) gene purchased from approved vendor, Integrated DNA Technologies (Coralville, IL, USA). The 2019-nCoV_N1-F primer (GAC-CCC-AAAATC-AGC-GAA-AT), the 2019-nCoV_N1-R primer (TCT-GGT-TAC-TGC-CAG-TTG-AAT-CTG), and the 2019nCoV_N1-P probe (FAM-ACC-CCG-CAT-TAC-GTT-TGG-TGG-ACC-BHQ1) sequences were all obtained directly from the US CDC website $[10,11]$. The RT-qPCR reaction was premixed following vendor guidelines and $1 \mu \mathrm{L}$ of lysate was added to bring the reaction to a final volume of $20 \mu \mathrm{L}$. The reaction was then loaded into the CFX Connect Real Time PCR Detection System (BioRad, Cat. No. 1855200) and run with the vendor recommended temperature and cycle timing for a total of 45 amplification cycles. Cq values were recorded and any sample with a Cq value less than or equal to 40 was labeled as positive for COVID-19 detection based on the US CDC recommended analysis of COVID-19 probe based RT-qPCR results $[10,11]$ (Figure 1, Table 1 ).

\begin{tabular}{|c|c|c|c|c|}
\hline Sample Number & Traditional Testing Status & Cq Value & Mean & Standard Deviation \\
\hline 1 & Positive & 24.93 & \multirow{20}{*}{29.94} & \multirow{20}{*}{5.44} \\
\hline 2 & Positive & 38.94 & & \\
\hline 3 & Positive & 37.25 & & \\
\hline 4 & Positive & 22.11 & & \\
\hline 5 & Positive & 36.57 & & \\
\hline 6 & Positive & 37.78 & & \\
\hline 7 & Positive & 29.18 & & \\
\hline 8 & Positive & 27.44 & & \\
\hline 9 & Positive & 32.05 & & \\
\hline 10 & Positive & 22.50 & & \\
\hline 11 & Positive & 31.33 & & \\
\hline 12 & Positive & 37.60 & & \\
\hline 13 & Positive & 28.78 & & \\
\hline 14 & Positive & 36.51 & & \\
\hline 15 & Positive & 22.58 & & \\
\hline 16 & Positive & 35.46 & & \\
\hline 17 & Positive & 34.70 & & \\
\hline 18 & Positive & 31.20 & & \\
\hline 19 & Positive & 31.26 & & \\
\hline 20 & Positive & $\mathrm{N} / \mathrm{A}$ & & \\
\hline
\end{tabular}




\begin{tabular}{|l|l|l|}
\hline 21 & Positive & 22.79 \\
\hline 22 & Positive & 24.37 \\
\hline 23 & Positive & 23.10 \\
\hline 24 & Positive & 24.15 \\
\hline 25 & Positive & 32.84 \\
\hline 26 & Positive & 26.11 \\
\hline 27 & Positive & 27.84 \\
\hline 28 & Positive & 29.04 \\
\hline
\end{tabular}

Table 1. RT-qPCR results following shaker-mill homogenization represented as Cq values from VTM containing patient samples that tested positive for COVID-19 when tested with the traditional extraction to PCR methodology. Only one sample, sample 20, was not detected as COVID-19 positive after shakermill homogenization processing from a previously confirmed COVID-19 positive patient. The 30 COVID19 negative samples are not shown because 0 of the 30 produced a Cq value following RT-qPCR.

Amplification

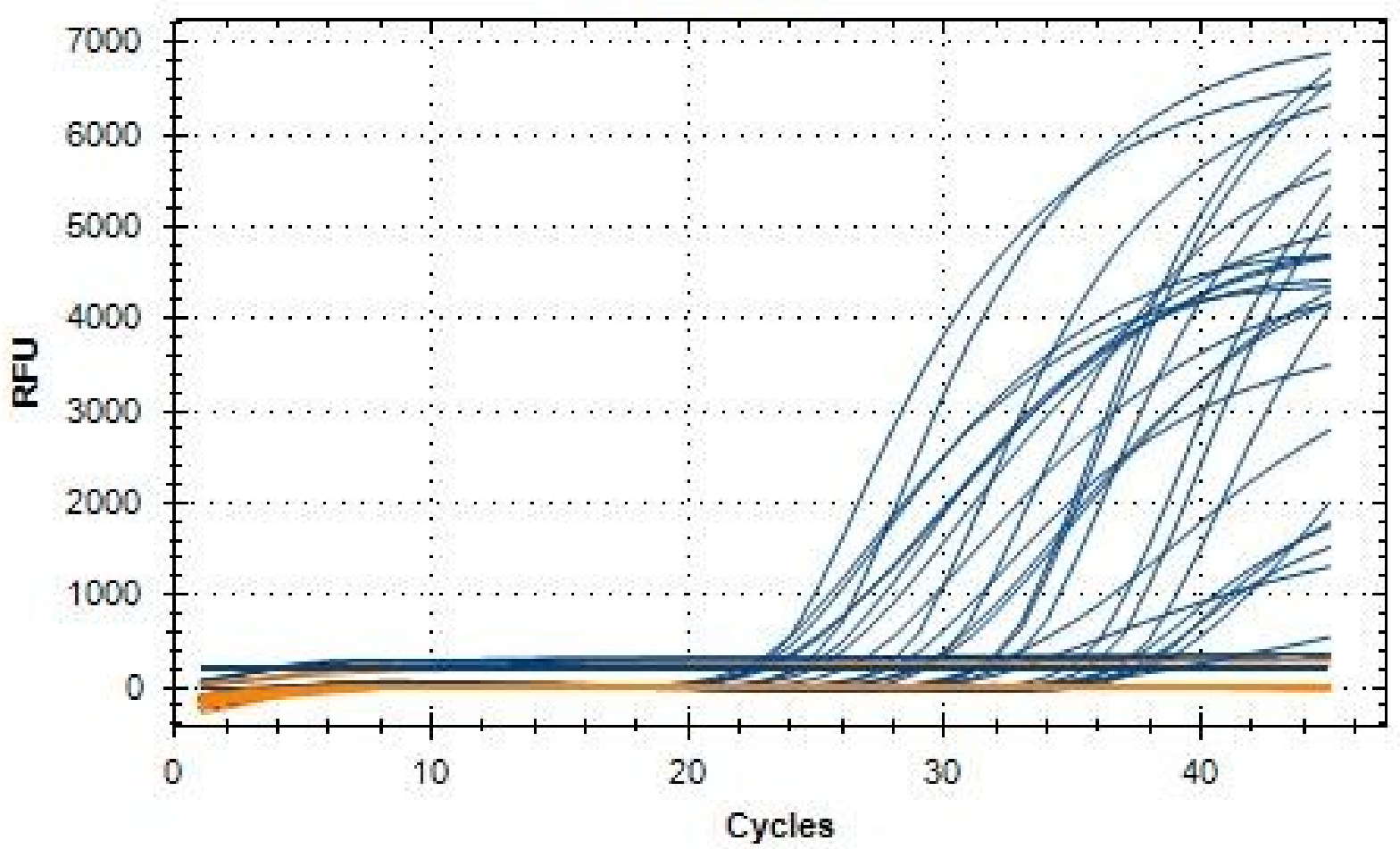

Figure 1. RT-qPCR results detecting the SARS-CoV-2 nucleocapsid gene off nasopharyngeal swabs processed via shaker-mill homogenization. Blue lines, VTM containing patient samples from COVID-19 positive patients processed via shaker-mill homogenization. Orange lines, VTM containing patient samples from COVID-19 negative patients processed via shaker-mill homogenization.

\subsection{Statistical Comparison of COVID-19 Detection Methodologies}


The positive or negative COVID-19 status of each sample as determined by the traditional processing method used by the Emory University laboratory was used as the baseline for our analysis of each sample. Following sample processing conducted via our novel methodology, we compared the positive or negative status obtained on each sample to the predetermined status in order to analyze the sensitivity and specificity when compared to the current extraction to PCR based testing model.

\section{Results}

With the use of shaker-mill homogenization on the Omni Bead Ruptor Elite, we were able to sufficiently lyse SARS-CoV-2 out of patient samples stored in VTM with the resulting lysate providing sufficient genetic material for RT-qPCR detection of the viral N gene. This data validates our novel two-step, direct-to-PCR approach for detecting COVID-19 off nasopharyngeal samples when run using the parameters described in this manuscript. With 27 of 28 COVID-19 positive samples being detected as positive following our methodology, and 0 of 30 COVID-10 negative samples detecting as positive, this detection method provides a $96.43 \%$ sensitivity and $100 \%$ specificity when compared to traditional extraction to PCR based methods (Figure 1, Table 1).

The 27 COVID-19 positive samples had an average Cq value of 29.94, with a standard deviation of 5.44 (Table 1). This average $\mathrm{Cq}$, falls 10 cycles below the US CDC cut-off Cq of 40 recommended for a COVID19 positive detection using these $N$ gene primers $[10,11,12]$. The wide standard deviation associated with these samples can be attributed to a variety of factors such as the variability of each patient's viral load, the quality of the swabbing procedure when obtaining each sample, and the inhibitors present in the VTM when going directly to RT-qPCR reactions [12]. This wide standard deviation in Cq values should not be viewed a prominent error in this methodology, but rather a byproduct of using patient samples, and should in no way diminish the statistical validity of the sensitivity and specificity reported for this novel process [12].

\section{Discussion}

Throughout the course of the COVID-19 pandemic, we have seen the need for efficient, effective, and timely viral testing procedures dramatically increase $[1,4]$. As the demand for these tests rise, the market availability of the reagents and consumable plastics required to complete the traditional extraction to PCR workflow have become increasingly scarce. This lack of availability in reagents and consumables needed for adequate testing is hindering the global efforts in disease surveillance and combatting of the SARS-CoV-2 pandemic. Herein, we believe our novel two-step, direct-to-PCR workflow is a potential solution to the resource pitfalls currently delaying testing in many parts of the world. While we acknowledge that this may not be the perfect solution for all instances, the authors feel that through bypassing the traditional extraction steps requiring multiple reagents and consumable plastics that this method has the potential to fill critical gaps in testing in resource challenged areas.

Additionally, with this validation using COVID-19 patient samples, resource challenged areas will be able to implement this technology with the reassurance that it has already proven a $100 \%$ positive predictive value and a $96.77 \%$ negative predictive value when compared to traditional extraction to PCR based methodologies in the first round of testing.

Aside from supplementing testing in resource challenged areas, this shaker-mill based detection workflow significantly reduces the time required to process each sample through bypassing the extraction steps, while increasing throughput of the process in preparing samples for PCR based 
detection. Using the Omni Bead Ruptor Elite, 24 swabs can be processed in 30 seconds, allowing for a full 384 well PCR plate to be prepared in as little as 30 minutes. This is in comparison to the hours of processing that a traditional viral RNA extraction kit requires [4,7]. As seen in the United States in July of 2020 when waiting periods for results after being swabbed have taken up to 8 days, any ability to reduce the processing time surrounding COVID-19 testing is currently of critical need in order to provide public health officials with the accurate data they need to advise patients and track infections $[1,2,4,7]$.

While additional, larger scale testing is still needed to gain a more robust statistical interpretation on the sensitivity and specificity of this methodology; we feel that after testing 58 sample resulting in a $96.43 \%$ sensitivity and $100 \%$ specificity, this technology is validated and viable for implementation in the arsenal of testing strategies currently employed surrounding COVID-19. It is our hope that this efficient and cost-effective measure for COVID-19 PCR based testing can work to fill the void in testing in many areas of the world or can be implemented for increasing the throughput of current testing sites, while reducing the time to obtain results.

\section{Author Contributions}

Conceptualization, Z.P.M, C.M.P., and R.J.N.; Methodology, Z.P.M. and C.M.P.; Validation, C.M.P., G.A.R., and R.J.N.; Writing - Original Draft Preparation, Z.P.M.; Writing - Review and Editing, Z.P.M., C.M.P, G.A.R., and R.J.N.; Supervision, R.J.N.; Funding Acquisition, Z.P.M. and R.J.N. All authors have read and agree to the published version of the manuscript.

\section{Funding}

This study was partially funded by the US National Institutes of Health project P30-AI050409 awarded to the Emory University Center for AIDS Research. Additional funding for this project was supported by private research funding from Omni International Inc (Kennesaw, GA, USA).

\section{Acknowledgments}

Firstly, we would like to acknowledge Colleen Kraft and Jessica Ingersoll of Emory University and the Emory Center for AIDS Research, the Emory University Clinical Virology Lab, and the Emory University School of Medicine for their assistance in procuring the COVID-19 patient samples used in this study. Additionally, we would like to acknowledge Pete Tortorelli, Karl Jahn, and Erik Masefield of Omni International Inc. for their financial support and organizational support of the experiments needed for this publication. Lastly, but certainly not least, we would like to acknowledge commitment and sacrifices of Rachel True, Rachel Nash, and Leah Proctor for their continued support of our work.

\section{Conflicts of Interest}

Z.P.M., C.M.P., G.A.R., and R.J.N. disclose that they are all currently employed by Omni International Inc in some capacity, however, none of the authors have any personal financial interests in the success or failure of this privately owned company. R.J.N. also discloses that he is the founder and CEO of JeevanBiosciences with personal financial interests in the success or failure of this privately owned company.

\section{References}


1. Tromberg BJ, Schwetz TA, Pérez-Stable EJ, et al. Rapid Scaling Up of Covid-19 Diagnostic Testing in the United States - The NIH RADx Initiative [published online ahead of print, 2020 Jul 22]. N Engl J Med. 2020;10.1056/NEJMsr2022263. doi:10.1056/NEJMsr2022263

2. Mishra DK, Goel G, Arora N, et al. The importance of intra- and inter-institutional networks for capacity building in severe acute respiratory syndrome coronavirus 2 reverse transcription polymerase chain reaction services: experience from an oncology centre in eastern India. Indian J Med Microbiol. 2020;38(1):9-17. doi:10.4103/ijmm.IJMM_20_255

3. Sung $\mathrm{H}$, Han MG, Yoo CK, et al. Nationwide External Quality Assessment of SARS-CoV-2 Molecular Testing, South Korea [published online ahead of print, $2020 \mathrm{Jul} 29$ ]. Emerg Infect Dis. 2020;26(10):10.3201/eid2610.202551. doi:10.3201/eid2610.202551

4. Ravi N, Cortade DL, Ng E, Wang SX. Diagnostics for SARS-CoV-2 detection: A comprehensive review of the FDA-EUA COVID-19 testing landscape [published online ahead of print, $2020 \mathrm{Jul}$ 18]. Biosens Bioelectron. 2020;165:112454. doi:10.1016/j.bios.2020.112454

5. Hashemi SA, Safamanesh S, Zadeh-Moghaddam HG, Ghafouri M, Amir A. High prevalence of SARS-CoV-2 and influenza A virus (H1N1) co-infection in dead patients in Northeastern Iran [published online ahead of print, 2020 Jul 28]. J Med Virol. 2020;10.1002/jmv.26364. doi:10.1002/jmv.26364

6. Won J, Lee S, Park M, et al. Development of a Laboratory-safe and Low-cost Detection Protocol for SARS-CoV-2 of the Coronavirus Disease 2019 (COVID-19). Exp Neurobiol. 2020;29(2):107119. doi:10.5607/en20009

7. Tang YW, Schmitz JE, Persing DH, Stratton CW. Laboratory Diagnosis of COVID-19: Current Issues and Challenges. J Clin Microbiol. 2020;58(6):e00512-20. Published 2020 May 26. doi:10.1128/JCM.00512-20

8. Lieberman-Cribbin W, Tuminello S, Flores RM, Taioli E. Disparities in COVID-19 Testing and Positivity in New York City [published online ahead of print, 2020 Jun 25]. Am J Prev Med. 2020;S0749-3797(20)30263-4. doi:10.1016/j.amepre.2020.06.005

9. Morehouse ZP, Proctor CM, Ryan GL, Nash RJ. A Novel Two-Step, Direct-to-PCR Method for Virus Detection off Swabs using Human Coronavirus 229E. Research Square; 2020. DOI: 10.21203/rs.3.rs-40525/v1.

10. Etievant S, Bal A, Escuret V, et al. Performance Assessment of SARS-CoV-2 PCR Assays Developed by WHO Referral Laboratories. J Clin Med. 2020;9(6):1871. Published 2020 Jun 16. doi:10.3390/jcm9061871

11. SoRelle JA, Frame I, Falcon A, et al. Clinical Validation of a SARS-CoV-2 Real-Time Reverse Transcription PCR Assay Targeting the Nucleocapsid Gene [published online ahead of print, 2020 Jun 1]. J Appl Lab Med. 2020;jfaa089. doi:10.1093/jalm/jfaa089

12. Broder K, Babiker A, Myers C, et al. Test Agreement between Roche Cobas 6800 and Cepheid GeneXpert Xpress SARS-CoV-2 Assays at High Cycle Threshold Ranges. J Clin Microbiol. 2020;58(8):e01187-20. Published 2020 Jul 23. doi:10.1128/JCM.01187-20 
